Vol. 70, N. ${ }^{\circ}$ III (mayo 20I8), 69-76

\title{
LA TEORÍA DE KARL MARX Y OTRAS TEORÍAS DEL VALOR: UTILIDAD, ESCASEZ Y OFERTA Y DEMANDA
}

\author{
DIEGO GUERRERO \\ Universidad Complutense de Madrid \\ Recepción de manuscrito: 12 de febrero de 2018 \\ Aceptación versión final: 13 abril de 2018
}

\begin{abstract}
RESUMEN En el artículo se compara la teoría laboral del valor desarrollada por Karl Marx con otras teorías del valor, haciéndolo de forma no exhaustiva, sino acotada a una selección de los conceptos más relevantes. El artículo se centra en tres de los conceptos que caracterizan a la teoría neoclásica del valor: la utilidad, la escasez y la interacción de la oferta y la demanda, concluyendo que las ideas de los críticos de la TLV no pueden servir para construir una alternativa a esta.
\end{abstract}

palabras ClAVE Teoría laboral del valor, Marx, neoclásicos, oferta y demanda, utilidad.

\begin{abstract}
The article compares Marx's labour theory of value with other theories of value, doing it in a non-exhaustive way but limited to a selection of the most relevant concepts. The article focuses on three of the concepts that characterize the neoclassical theory of value: utility, scarcity and the market interaction of demand and supply, to conclude that the ideas of the critics of the LTV are unable to build an alternative to the latter.
\end{abstract}

KEYWORDS Labour theory of value, Marx, neoclassicists, supply and demand, utility.

JEL CODES B12, B13, B14.

\section{INTRODUCCIÓN}

En este artículo se critican varias de las ideas sobre las que los autores neoclásicos pretendieron construir una teoría alternativa a la teoría laboral del valor (TLV), principalmente la escasez, la utilidad y la oferta y la demanda. Al repasar las críticas de estos conceptos hechas por los clásicos, Cournot (1838), Marx y su discípulo Rubin (1923), se pretende mostrar por qué la teoría neoclásica no puede servir de alternativa a la TLv.

\section{UTILIDAD Y ESCASEZ EN LOS PRIMEROS NEOCLÁSICOS}

Aunque existen numerosos antecedentes del «triunvirato» Jevons-Menger-Walras - por ejemplo, von Thünen, Dupuit o Gossen -, la teoría de la utilidad marginal se asocia habitualmente 
con los primeros, quienes protagonizaron en la década de 1870 una «revolución» basada en la idea de la utilidad marginal, que, más allá de su importancia en sí misma, pretendía derribar la TLV. Por ejemplo, W. S. Jevons resume su teoría de la utilidad marginal como el reverso de la teoría laboral:

La reiterada reflexión y la investigación me han conducido a la en cierto modo novedosa opinión de que el valor depende enteramente de la utilidad. Las opiniones dominantes consideran el trabajo antes que la utilidad como el origen del valor. $Y$ hay incluso quienes sostienen de forma inequívoca que el trabajo es la causa del valor. Yo muestro, por el contrario, que basta con perfilar cuidadosamente las leyes de la variación de la utilidad como dependiente de la cantidad de mercancía en nuestro poder, para llegar a una teoría del intercambio satisfactoria, de la cual las leyes ordinarias de la oferta y la demanda son una consecuencia necesaria. (Jevons 1871, pp. 67-68)

Algo similar ocurre con Carl Menger, quien señala:

Respecto del valor de un diamante, es indiferente que haya sido descubierto por puro azar o que se hayan empleado mil días de duros trabajos en un pozo diamantífero. [...] Y así, no pocas veces, bienes en los que se ha empleado mucho trabajo no tienen ningún valor y otros en los que no se ha empleado ninguno lo tienen muy grande. (Menger, 1871, p. 132)

Jevons señala que en su teoría la «ley general» consiste en "que el grado de utilidad varía con la cantidad de mercancía y, en última instancia, disminuye a medida que esa cantidad aumenta», y la atribuye, entre otros, a Dupuit y a Gossen (Jevons, 1871, p. 50). Pero este autor habla también con admiración de la obra de Cournot (1838), a pesar de que éste:

No elaboró ninguna teoría definitiva del ámbito y la naturaleza de la utilidad y el valor, sino que, partiendo de los hechos palpables conocidos referentes a las relaciones de precio, producción y consumo de mercancías, investigó estas relaciones analítica y diagramáticamente con una fuerza y un acierto que nada dejan que desear. Esta obra debe ocupar un lugar notable en la historia de la disciplina. (Jevons, 1871, pp. 50-51)

En realidad, Jevons no hace justicia a Cournot, pues no solo no hay en él ni rastro de cualquier teoría de la utilidad, sino que enfatiza la superioridad de la TLV — que acepta de forma tácitasobre los débiles fundamentos de cualquier teoría que ligue el valor con la utilidad. Cournot distingue, como Ricardo y posteriormente Marx, entre valor y riqueza — solo que llama a esta última «riqueza en sentido concreto», y al valor «riqueza en sentido abstracto»— y contrapone los sólidos fundamentos objetivos del «valor de cambio»—que en su época no pueden ser sino el trabajo - con todo lo relativo a la utilidad y la escasez:

Hay que distinguir bien entra la idea abstracta de riqueza o de valor de cambio, idea fija, susceptible por consiguiente de prestarse a combinaciones rigurosas, y las ideas accesorias de utilidad, rareza, aptitud para la satisfacción de las necesidades y gozos humanos, que todavía despierta en el lenguaje ordinario la palabra riqueza: ideas variables e indeterminadas por naturaleza, sobre las cuales no se podría asentar una teoría científica. (Cournot, 1838, p. 25) 
Por su parte, Walras defiende una teoría basada en la rareté —o escasez — que rechaza tanto la «solución inglesa» - ligada al trabajo y a los nombres de Adam Smith y David Ricardocomo la «francesa» - ligada a la utilidad y a Condillac y J.-B. Say- (Walras, 1874, p. 336). Para ello, parte de la «riqueza social» como «conjunto de cosas materiales o inmateriales [...] que son escasas, es decir, que por una parte nos son útiles y, por otra, existen a nuestra disposición en cantidades limitadas» (ibíd., pp. 155-156); estas cosas son «apropiables», «intercambiables» e «industrialmente producibles o multiplicables», y su rareté se presenta "como la intensidad de la última necesidad que es o podría haber sido satisfecha» por ellas, definida «en términos de la doble condición de utilidad y limitación en la cantidad» (ibíd., pp. 155-57, 248). Mientras que «el valor de cambio es real u objetivo», la «rareté es personal o subjetiva» e igual a «la derivada de la utilidad efectiva respecto a la cantidad poseída» (ibíd., p. 250).

Sorprende mucho que Walras cite a Smith y no a Ricardo — ni a Marx, aunque esto sería más fácil de entender - para ilustrar la TLV, teoría esta — que «es, más que una teoría demasiado estrecha, una teoría completamente vacía, más que una afirmación inexacta, una afirmación gratuita» - de la que dice que «ha sido generalmente mal refutada», pues no se ha usado el argumento básico correcto, a saber, que «si el trabajo tiene valor y se intercambia es porque es a la vez útil y limitado en su cantidad, es decir porque es escaso» (Walras, 1874, p. 337).

De la crítica a la escuela «inglesa» pasa Walras a la «francesa», acusando a la teoría «de la utilidad» — como algo distinto de su rareté- de ser demasiado «amplia», pues la utilidad «no es suficiente para crear valor: es preciso además de que la cosa sea útil, que no exista en cantidad ilimitada, que sea escasa» (Walras, 1874, p. 338).

Podemos concluir, por tanto, que al insistir en los dos elementos, utilidad y escasez, lo que está haciendo Walras no es otra cosa que reivindicar la simetría de la oferta y la demanda, ya que la escasez se refiere al esfuerzo, al trabajo o al coste de producción, y esto es lo que los neoclásicos ven tras la curva de oferta. En el epígrafe siguiente, enfrentaremos precisamente esta tesis de la simetría con la tesis opuesta de la asimetría, más conforme con la TLV.

\section{LA OFERTA Y LA DEMANDA}

A primera vista, la idea de que los precios de las mercancías vienen determinados por la interacción entre oferta y demanda parece sensata, y se ha atribuido a Aristóteles y sus discípulos medievales pero también a autores modernos de la época de la producción capitalista. Sin embargo, desde que se elaboraron las primeras teorías científicas del valor, diversos autores de diferentes escuelas de pensamiento — desde Ricardo y Marx a Böhm-Bawerk o Sraffa— han mostrado las limitaciones de dicha idea y comprendido la necesidad de postular unos fundamentos diferentes, más profundos, del valor de las mercancías.

\section{LOS CLÁSICOS}

La utilización de la demanda en el análisis microeconómico no exige compartir las teorías neoclásicas sobre sus determinantes: la utilidad marginal. De hecho, el análisis de la demanda se puede llevar a cabo con independencia de la utilidad marginal, y así se ha hecho históricamente antes y después del viraje marginal-subjetivista de finales del siglo XIX. Los clásicos 
estaban al corriente de la «ley de la demanda» y Adam Smith tenía una noción muy clara de qué significa para los precios la existencia de escasez o excedente en un mercado. Smith conoce la ley del precio único y expone las diversas posiciones que puede presentar el precio de mercado en relación con el «precio natural», ese "precio central, alrededor del cual gravitan continuamente los precios de todas las mercancías», teniendo en cuenta que «contingencias diversas pueden a veces mantenerlos suspendidos, durante cierto tiempo, por encima o por debajo de aquél; pero, cualesquiera que sean los obstáculos que les impiden alcanzar su centro de reposo y permanencia, continuamente gravitan hacia él» (Smith, 1776, p. 57).

David Ricardo dedica el capítulo xxx de su libro a «la influencia de la demanda y de la oferta sobre los precios», defendiendo «la opinión de que el precio de las cosas depende exclusivamente de la proporción existente entre la demanda y la oferta», tesis que "se ha convertido casi en un axioma en Economía política y ha sido fuente de muchos errores en dicha ciencia» (Ricardo, 1817, p. 385). De hecho,

[E]s el coste de la producción el que debe regular en último término el precio de las cosas, y no, como se ha dicho a menudo, la proporción existente entre la oferta y la demanda: ésta puede, en verdad, afectar durante algún tiempo el precio de mercado de un artículo, hasta que la oferta de éste sea más o menos abundante, según que la demanda haya aumentado o disminuido; pero este efecto será sólo de duración temporal. (Ricardo, 1817, p. 385)

Es decir, Ricardo, como Smith, argumenta contra el principio de la oferta y la demanda y defiende la tesis de la asimetría: el efecto de una variación de la demanda sobre el precio solo puede ser «temporal» - salvo que varíe el coste de producción-, mientras que una variación del coste puede generar una variación equivalente del precio sin que la oferta y la demanda se hayan alterado.

Y John Stuart Mill recapitula así las ideas de Smith y Ricardo:

La demanda y la oferta rigen el valor de todas las cosas cuya cantidad no puede aumentarse indefinidamente; solo que, aun para ellas, cuando son producto de la actividad humana, existe un valor mínimo fijado por el costo de producción. Pero en todos los casos en que pueden multiplicarse al infinito, la demanda y la oferta solo determinan las perturbaciones del valor durante un periodo que no puede exceder del tiempo necesario para que se altere la oferta. Así, pues, mientras regulan las oscilaciones del valor, ambas obedecen a una fuerza superior, que hace que $e l$ valor gravite hacia el costo de producción, la cual lo fijaría y lo mantendría ahí si no surgieran continuamente nuevas influencias perturbadoras que la hacen desviarse otra vez. Siguiendo la misma línea de metáfora, la demanda y la oferta tienden siempre hacia un equilibrio, pero la situación de equilibrio estable se alcanza cuando las cosas se cambian unas por otras de acuerdo con su coste de producción, o, según la expresión que hemos usado, cuando las cosas están a su valor natural. (Mill, 1848, p. 402) [Cursivas añadidas por el autor del artículo]

\section{COURNOT}

Como sabemos, A. A. Cournot ocupa una posición muy especial en la historia del pensamiento económico, ya que se trata de un marginalista — que aplicó el cálculo diferencial al estudio de los problemas económicos - que no es un utilitarista - sino defensor de la teoría del valor de 
los clásicos-, y, por tanto, «reticente a conectar su función de demanda con un elemento tan controvertido como la utilidad» (Georgescu-Roegen, 1983, p. LVXvi).

El bagaje «ricardiano» de Cournot se refleja en la manera como registra las diferencias entre la evolución de los valores y de las riquezas. Así, pone el ejemplo del librero Dupont, quien, al sacrificar una parte de los ejemplares de una obra rara, destruyéndolos, ha podido obtener «más beneficio de la parte restante que de la edición completa»; o de la Compañía Holandesa, que «destruía en las islas de la Sonda una parte de las valiosas especias que monopolizaba» por la misma razón (Cournot, 1838, p. 26): ambos ejemplos muestran que «esta destrucción material es una verdadera creación de riqueza en el sentido comercial de la palabra», tanto desde un punto de vista individual — «el inventario del librero recibirá justamente la existencia de un valor mayor en su activo» - como social, ya que en «el inventario general o balance de la riqueza en circulación, encontraríamos un acrecentamiento en la suma de esta riqueza» (ibíd., pp. 26-27). Simétricamente, se puede destruir riqueza comercial por medio de la creación de nuevas mercancías. ${ }^{1}$

\section{MARX}

El planteamiento de Marx en relación con el papel de la oferta y la demanda en la teoría del valor coincide básicamente con el de los clásicos, si bien la «ley» de la oferta y la demanda le parece «incuestionable» (Marx, 1885, p. 536). Su explicación de los movimientos de la oferta y la demanda es de una claridad meridiana: si la demanda cae, los precios bajan, y «a consecuencia de ello se retirará capital de la producción de estos artículos hasta que su oferta se reduzca a la medida que corresponde a su papel modificado en el proceso social de producción», de forma que «reducirse así su producción, subirán nuevamente los precios al nivel normal, siempre que el valor no se modifique por otras causas» (ibídem). Pero ese nivel normal del precio no es sino el valor —el «valor de mercado» o sectorial, como media ponderada de los valores «individuales»—, de forma que si se puede decir que la oferta y demanda regulan «las desviaciones de los precios de mercado con respecto al valor de mercado», también es cierto que «el valor de mercado regula la relación entre oferta y demanda o el centro en torno al cual las fluctuaciones de la oferta y la demanda hacen oscilar, a su vez, los precios de mercado» (Marx, 1894, p. 229).

Marx señala que las desigualdades entre la oferta y la demanda son fáciles de comprender, mientras que «la dificultad real estriba en definir qué debe entenderse por coincidencia entre la oferta y la demanda» (Marx, 1894, p. 239), ya que en este caso, cuando ambas se anulan mutuamente, «dejan de explicar nada», y entonces el precio es el «precio determinado independientemente de la relación entre la oferta y la demanda, es decir, su precio natural», que es «el objeto que realmente había que analizar» (ibíd.; y Marx, 1867, pp. 654-655). En realidad, el «precio natural» de los clásicos, desarrollo del «precio necesario» de los fisiócratas, se convierte en Marx en el «precio de producción», por lo que la idea anterior se expresa con mayor propiedad en el libro iII de El capital:

Si coinciden la oferta y la demanda, el precio de mercado de la mercancía corresponde a su precio de producción, es decir, que su precio aparece entonces regulado por las leyes internas de la producción capitalista, independientemente de la competencia. (Marx, 1894, p. 454) 
La gran diferencia entre este enfoque y el «neoclásico» es similar a las diferencias entre Marx y Malthus, quien pretendía demostrar que «la gran ley de la demanda y la oferta entra en acción para determinar lo que Adam Smith llama precios naturales y también lo que el mismo autor denomina precios de mercado», de forma que el principio de la oferta y la demanda sería «el dominante» frente al principio alternativo de los costes de producción, que solo sería «secundario», como correspondería al hecho de que los costes sólo son una «condición necesaria de la oferta de los objetos deseados» (Malthus, 1820, pp. 6o-62). Pero Marx le recuerda a Malthus que:

Puede haber dos precios naturales sumamente diferentes, en diferentes momentos, para una misma mercancía, y no obstante la proporción que guarda la oferta para con la demanda puede ser, en ambos casos, la misma, a saber, la proporción de igualdad. Se admite, pues, que en el caso de dos natural prices de una misma mercancía en diferentes momentos, la oferta y la demanda pueden y deben coincidir en ambos casos si la mercancía ha de venderse en ambas oportunidades a su natural price. Pero puesto que en ninguno de ambos casos hay diferencia alguna en la proporción entre oferta y demanda, pero sí la hay en la magnitud del propio natural price, éste obviamente se determina en forma independiente de la oferta y la demanda, y de ninguna manera puede ser determinado por éstas. (Marx, 1894, p. 243)

En Marx, como en los clásicos, en Cournot y en casi todos los autores anteriores a la corriente subjetivista neoclásica, la demanda está determinada, pues, por circunstancias que no tienen nada que ver con la utilidad marginal.

\section{RUBIN}

Para disolver las dudas suscitadas por Malthus, el marxista ruso I. I. Rubin analizó el caso de la producción con costes crecientes. Aparentemente, como en este caso un aumento de la demanda provocaría un aumento de la producción y también del coste de producción medio, parecen tener razón quienes coinciden con Malthus en que «la demanda determina de alguna manera el valor»; pero Rubin argumenta que «los defensores de este argumento olvidan una circunstancia muy importante», a saber: que «en el ejemplo que examinamos, las variaciones en el volumen de la producción significan al mismo tiempo variaciones de las condiciones técnicas de producción dentro de la misma rama» (Rubin, 1923, p. 263). Por eso, tras examinar diferentes casos en los que la producción que se lleva a cabo por varios grupos de empresas de diferente eficiencia da lugar a diferentes valores del producto, concluye afirmando que:

El valor ha cambiado precisamente porque las condiciones de producción cambiaron en dicha rama. De este ejemplo no debemos extraer la conclusión de que los cambios en el valor están determinados por cambios en la demanda y no por cambios en las condiciones técnicas de producción. Por el contrario, la conclusión solo puede ser que los cambios en la demanda no pueden influir en la magnitud del valor de ningún modo excepto modificando las condiciones técnicas de producción en la rama considerada. (Ibídem) [Cursivas añadidas por D. Guerrero]

Así pues, la proposición básica de la TLV sigue siendo válida, y el que la demanda pueda influir sobre el valor indirectamente no contradice de ninguna manera a la TLV. En el desarrollo de las fuerzas productivas influye una serie de condiciones sociales, políticas y hasta culturales, pero: 
Variadas condiciones económicas y sociales, que incluyen las variaciones de la demanda, pueden afectar al valor, no junto a las condiciones técnicas de la producción, sino solamente mediante cambios en las condiciones técnicas de producción. Así, la técnica de producción sigue siendo el único factor que determina el valor. (Rubin, 1923, p. 266)

Vemos, pues, que los autores estudiados coinciden en que, en el análisis del equilibrio de mercado, son las condiciones de producción - la oferta - las que determinan los precios de equilibrio - tesis de la asimetría - en contra de la tesis implícita en la metáfora marshalliana de «las tijeras» (Marshall, 1890, p. 197): el presunto juego simétrico de la oferta y la demanda.

\section{CONCLUSIONES}

Los autores neoclásicos, críticos con la TLV de Marx, propusieron y proponen como alternativa, no una sino varias teorías del valor, o al menos varios conceptos usados en su intento de construir una teoría alternativa: la utilidad marginal, la oferta y demanda, y la escasez. En este artículo, hemos pasado revista y criticado esos conceptos.

Contra lo que normalmente se hace, no se puede identificar a marginalistas y partidarios de la utilidad marginal; por eso es tan importante la figura de Cournot, quien fue quizás el primer marginalista, defensor de una teoría del valor ricardiana, coincidente en lo esencial con la teoría de Marx, y opuesto a cualquier consideración de la utilidad en la teoría del valor.

Por otra parte, la TLV de Marx es perfectamente compatible con la ley de la demanda, o relación inversa entre precio y cantidad demandada, que es completamente independiente de la utilidad; además, no niega el juego de la oferta y la demanda en la determinación de los equilibrios a corto plazo del mercado, pero sí que niega ese papel en la determinación del precio «natural»—-término de Smith coincidente con el «precio de producción» de Marx-. Marx muestra que el precio de producción, que a su vez viene determinado por el valor-trabajo de la mercancía, es el que regula el juego de la oferta y la demanda, y muestra que el papel de la demanda queda reducido a su cooperación, junto a la oferta, en la determinación de la cantidad demandada — no el precio - de equilibrio. En este aspecto, también la aportación de Rubin fue muy importante en el desarrollo de la tesis de la «asimetría».

De esta manera, podemos concluir que la superioridad de la TLV frente a la teoría neoclásica del valor es un hecho difícil de ocultar.

\section{NOTA}

1 Supongamos que la rareza de un libro determinado permite vender a 300 francos cada uno de los 50 ejemplares existentes; si un «librero reimprime mil ejemplares de este libro, que valdrán 5 francos», esto producirá una disminución del valor de cambio total: «He aquí una operación industrial, una producción material, útil al librero que la emprende, útil a todos cuantos le han abastecido de materiales y servicios, útil también al público, por escasas enseñanzas de provecho que encierre el libro, pero que constituye una verdadera destrucción de riqueza en el sentido abstracto y comercial de la palabra» (Cournot, 1838, p. 27). 


\section{REFERENCIAS}

Cournot, A. A. (1838). Investigaciones acerca de los principios matemáticos de la teoría de las riquezas, Madrid, España: Alianza, 1969.

Georgescu-Roegen, N. (1983 [1854]). Introduction. In H. Gossen, The laws of human relations and the rules of human action derived therefrom (pp. XI-CXLV). Cambridge, EE. UU.: MIT Press.

Jevons, W. S. (1871). La teoría de la economía política. Madrid, España: Pirámide, 1998.

Malthus, T. R. (1977 [1820]). Principios de Economía Política. México DF, México: Fondo de Cultura Económica.

Marshall, A. (1890). Principles of Economics. London, England: Pantianos Classics.

Marx, K. (1867). El capital. Crítica de la Economía Política. Libro I. Madrid, España: Siglo XXI, 1978.

Marx, K. (1885). El capital. Crítica de la Economía Política. Libro II. Madrid, España: Siglo xxi, 1979.

Marx, K. (1894). El capital. Crítica de la Economía Política. Libro III. Madrid, España: Siglo Xxi, 1979.

Menger, C. (1983 [1871]). Principios de economía política. Madrid, España: Unión Editorial.

Mill, J. S. (1951 [1848]). Principios de economía política con algunas de sus aplicaciones a la filosofía social. México DF, México: Fondo de Cultura Económica.

Ricardo, D. (1973 [1817]). Principios de economía política y tributación. Madrid, España: Ayuso.

Rubin, I. I. (1974 [1923]). Ensayo sobre la teoría marxista del valor. Buenos Aires, Argentina: Pasado y Presente.

Smith, A. (1980 [1776]). Investigación sobre la naturaleza y causas de la riqueza de las naciones. México DF, México: Fondo de Cultura Económica.

Walras, L. (1987 [1874]). Elementos de Economía política pura (o Teoría de la riqueza social). Madrid, España: Alianza. 